\title{
BMJ Open Understanding transitional care programmes for older adults who experience delayed discharge: a scoping review protocol
}

\author{
Katherine S McGilton (D) , ${ }^{1,2}$ Shirin Vellani (D) ,1,2 Jessica Babineau, ${ }^{1}$ \\ Jennifer Bethell (D) , ${ }^{1}$ Susan E Bronskill, ${ }^{3}$ Elaine Burr, ${ }^{4}$ Margaret Keatings, ${ }^{1}$ \\ Janet E McElhaney, ${ }^{5}$ Sandra McKay, ${ }^{6}$ Kathryn Nichol, ${ }^{7}$ Abeer Omar, ${ }^{1}$ \\ Martine T E Puts, ${ }^{2}$ Anita Singh, ${ }^{8}$ Laura Tamblyn Watts, ${ }^{9}$ Walter P Wodchis (D),${ }^{10}$ \\ Souraya Sidani (1) ${ }^{11}$
}

To cite: McGilton KS, Vellani S, Babineau J, et al. Understanding transitional care programmes for older adults who experience delayed discharge: a scoping review protocol. BMJ Open 2019;9:e032149. doi:10.1136/ bmjopen-2019-032149

- Prepublication history and additional material for this paper are available online. To view these files, please visit the journal online (http://dx.doi. org/10.1136/bmjopen-2019032149).

Received 05 June 2019 Revised 04 November 2019 Accepted 21 November 2019

Check for updates

(C) Author(s) (or their employer(s)) 2019. Re-use permitted under CC BY-NC. No commercial re-use. See rights and permissions. Published by BMJ.

For numbered affiliations see end of article.

Correspondence to Dr Katherine S McGilton; kathy.mcgilton@uhn.ca

\section{ABSTRACT}

Introduction Many hospitalised older adults experience delayed discharges due to increased postacute health and social support needs. Transitional care programmes (TCPs) provide short-term care to these patients to prepare them for transfer to nursing homes or back to the community with supports. There are knowledge gaps related to the development, implementation and evaluation of TCPs. The aims of this scoping review (ScR) are to identify the characteristics of older patients served by TCPs; criteria for transfer, components and services provided by TCPs; and outcomes used to evaluate TCPs.

Methods and analysis The study involves six-step $\mathrm{ScR}$ and is informed by a collaborative/participatory approach whereby stakeholders engage in the development of the research questions, identification of literature, data abstraction and synthesis; and participation in consultation workshop. The search for scientific literature will be done in the Medline, PsychINFO, Emcare and CINAHL databases; as well, policies and reports that examined models of transitional care and the outcomes used to evaluate them will be reviewed. Records will be selected if they involve community dwelling older adults aged 65 years or older, or indigenous persons 45 years or older; and presented in English, French, Dutch and German languages. Records will be screened, reviewed and abstracted by two independent reviewers. Extracted data will be analysed using descriptive statistics and a narrative analysis, and organised according to Donabedian's model of structure (characteristics of older adults experiencing delayed discharge and served by TCPS), process (TCP components and services) and outcome.

Ethics and dissemination This ScR does not require ethics approval. Dissemination activities include integrated knowledge translation (KT) (consultation with stakeholders throughout the study) and end-of-grant KT strategies (presentations at national and international conferences; and publication in peer-reviewed interdisciplinary journal).
Strengths and limitations of this study

The study takes a collaborative and participatory ap proach involving multidisciplinary and multi-sectoral partners also including family caregivers.

- It will include both scientific and grey literature in English, Dutch, German and French languages.

- The study will only synthesise data related to older adults who experience delayed discharge and are served by transitional care programmes.

\section{INTRODUCTION}

Many older adults live with multiple complex health issues, compounded by risk factors related to social determinants of health that adversely affect their overall quality of life. ${ }^{1}$ Due to an exacerbation of these conditions or other acute events such as falls, older adults are often admitted to tertiary care hospitals. ${ }^{2}$ However, when there are insufficient health and social supports to meet their postacute care needs, many hospitalised older adults cannot be discharged once treatments are completed. ${ }^{2}$ In a recent systematic review of 64 studies, Landeiro and colleagues estimated that on average, $29 \%$ of hospitalised older adults experience delayed discharges. ${ }^{3}$ Delayed discharges from acute care hospitals is a global phenomenon, ${ }^{3}$ and have presented a critical challenge for many healthcare systems for several decades. ${ }^{245}$ In Canada, patients who experience delayed discharges, but no longer require the intensity of services provided in hospitals, are designated as 'alternate level of care' (ALC). ${ }^{6}$ In Ontario, the proportion of hospital beds occupied by ALC patients reached $14.8 \%$ in 2016-2017. ${ }^{7}$

Delayed discharges significantly reduce patient flow, leading to emergency department 
overcrowding, cancellation of outpatient elective surgeries, and poor coordination of community care resources. ${ }^{38} \mathrm{In}$ addition to systemic challenges, delayed discharges also lead to psychological distress in older adults and their care partners, presented in the form of depressive, ${ }^{8}$ anxiety and stress symptoms. ${ }^{9}{ }^{10}$ Furthermore, while waiting for an appropriate discharge location, older adults may experience generalised deconditioning and iatrogenic complications, such as poor nutritional status, delirium, falls and infection; as well as social isolation. ${ }^{3}{ }^{11}$ In addition, some hospitals charge a daily copayment to patients with prolonged delayed discharge, which may place a substantial financial burden on some. ${ }^{10}$

The lack of appropriate community care resources contributes to the system-wide issue of delayed discharges, preventing older adults from getting the right care, at the right time, and in the right setting. Transitional care programmes (TCPs) have been developed to provide shortterm, low intensity, restorative care to these patients in a variety of settings such as hospitals, nursing homes or other assisted living facilities. ${ }^{8}$ TCP are meant to enhance function of older adults by providing health and social care prior to their return to a final destination such as nursing home or back to the community with supports. In turn, TCPs address the individual and health system impact of delayed discharges. ${ }^{12}{ }^{13}$ Current descriptions and reported effectiveness vary across healthcare contexts and countries, making it difficult for clinical and policy decision makers to determine which TCP model works, for whom, and what support is required for their implementation.

Our preliminary discussions with stakeholders (including clinical and policy decision makers, as well as caregivers of patients admitted to TCPs in Ontario) have identified knowledge gaps about how to best develop, implement and evaluate TCPs, including the patients they should serve, the resources they require, the services they should deliver and the methods that should be used to evaluate them. In this paper, we set out the protocol for a scoping review $(\mathrm{ScR})$ to examine the literature on TCPs to identify knowledge required to support older adults who no longer require acute care services, but who are unable to transition directly home. Specifically, this review seeks to answer the following four research questions:

1. What are the characteristics of older patients served by TCPs?

2. What are the criteria used to transfer patients from hospital to TCPs, and from TCPs to other care locations?

3. What care components and services are provided by TCPs?

4. What patient, caregiver and health system outcomes have been used to evaluate TCPs?

\section{METHODS AND ANALYSIS}

A ScR is most suited to address our questions because it is 'a form of knowledge synthesis that addresses an exploratory research question aimed at mapping key concepts, types of evidence, and gaps in research related to a defined area of study or clinical practice by systematically searching, selecting and synthesizing existing knowledge'. ${ }^{14}$ ScRs can include varied types of studies, policy documents and grey literature that are typically excluded from systematic reviews. We will follow the ScR methodology framework outlined by Arksey and O'Malley ${ }^{15}$ and refined by Levac et al, ${ }^{16}$ Colquhoun $e t a l,{ }^{14}$ and Daudt et $a l,{ }^{17}$ and the Preferred Reporting Items for Systematic Review and Meta-Analysis Protocols extension for ScRs statement (see online supplementary appendix A). ${ }^{18}$ The ScR framework includes six steps.

\section{Step 1: identify research questions}

In collaboration with our stakeholders, we identified four research questions, listed previously.

\section{Step 2: identify relevant literature}

As per recommendations for ScRs, our search will be as comprehensive as possible, covering both scientific and grey literature. ${ }^{14-17}$ Based on the aims of the review, following key words and Medical Subject Headings terms were included in the search: aged, indigenous persons and TCP. A comprehensive search strategy was developed in conjunction with a health sciences librarian on our team and subsequently refined in consultation with stakeholders and the research team. The strategy was validated by ensuring the retrieval of a key set of relevant studies. We searched OVID Medline, Embase, PsychINFO and Emcare; as well as EBSCO CINAHL. Final searches were completed in May 2019, and we anticipate completion of the review processes by December 2019. For full Medline search strategy, see online supplementary appendix B. In addition to bibliographic database searching, we will conduct a thorough search of grey literature such as community and policy reports, government or public agency publications, and practice guidelines. This will be done by searching Google Advanced and websites of both government and private agencies that fund, report and evaluate TCPs. We will also review the reference lists of included studies and consult stakeholders and experts to help identify additional literature. Bibliographic information will be managed using EndNote X8. Systematic de-duplication will be carried out using the Bramer method for Endnote users. ${ }^{19}$ Records will be imported into Covidence, a web-based application for screening and data extraction. ${ }^{20}$

\section{Patient and public involvement}

This ScR study does not involve patient participation. However, our stakeholders include family caregivers, who are involved in the development of the research questions, identification of literature, data extraction and synthesis; and participation in consultation workshop.

\section{Step 3: study selection}

\section{Inclusion criteria}

We will include all published and unpublished literature reporting any quantitative, qualitative, mixed or multimethod research, as well as policies and reports related to the TCPs. Articles in English, French, Dutch and German 
languages will be included. Acknowledging that persons from indigenous communities experience complex health conditions at younger ages than others, ${ }^{21-23}$ we will include literature involving community dwelling older adults 65 years of age or older, or indigenous persons 45 years or older, ${ }^{22}$ so as to acquire relevant literature related to the care needs of ageing indigenous persons. ${ }^{24}$ We will include all literature that examined models of transitional care and the outcomes used to evaluate them.

We will use the a priori inclusion criteria to screen the citations by two independent reviewers. First, we will screen the titles and abstracts, followed by full-text review of the selected articles using Covidence. To ensure reliability between reviewers, we will conduct training before commencing screening, followed by weekly teleconference to discuss any ongoing concerns. In case of disagreement between the reviewers in the selection process, other team members will be consulted to form a consensus.

\section{Step 4: data extraction}

All studies and reports that meet inclusion criteria will be charted by two independent reviewers using Covidence. The data extraction form will be piloted on five studies, to ensure consistency. As described by Arksey and O'Malley, charting is an iterative process, ${ }^{15}$ therefore, any changes to the form deemed necessary will be made in consultation with the team prior to the extraction of all articles. Data to be extracted include: publication type (eg, journal article or grey literature), study type (eg, quantitative, qualitative, or mixed), study characteristics/setting, TCP patient characteristics (eg, demographic and clinical characteristics), models of TCP (eg, components and services provided), criteria and strategies used to transfer patients across care locations, transitional care practice guidelines and types of healthcare providers involved in the TCP. We will also extract patient, caregiver and health system outcomes, barriers and facilitators to, as well as challenges in, providing effective transitional care services.

\section{Step 5: synthesis and presentation of results}

The results of the current review will be presented in the context of each objective, using two strategies: (1) a numerical overview of the amount and type of the included literature; and (2) a narrative synthesis and mapping of the results. ${ }^{141617}$ The narrative mapping exercise will be foundational for generating a framework to inform the implementation and evaluation of TCPs. The framework will be organised into three sections, consistent with Donabedian's structure-process-outcome model. ${ }^{25}$ We define structures as characteristics that are within the healthcare team, organisation, or patient that influence the design, implementation and/or effectiveness of TCPs. Examples of such characteristics are health or clinical characteristics of patients admitted to TCPs; and the material and human resources needed for the implementation of TCPs. Processes refer to the components of care (eg, comprehensive geriatric assessment) provided to patients admitted to TCPs; healthcare team members' practices (eg, case conferences, inter-professional communication); and patients' behaviours (eg, engagement in self-care) or actions with their surroundings, including team members. Outcomes involve any specified indicators of quality of care for the patients (eg, improved physical function), caregivers (eg, reduced burden), and the healthcare system (eg, patient flow across settings, mortality) in the context of TCPs.

We will summarise the various types of TCPs identified in the literature that are established to address the concern related to delayed hospital discharges. We will also describe and summarise the results of evaluation of different TCPs that are reported in grey literature. If the data are available, our analyses will attempt to identify the effects of sex (ie, biological attributes) and gender (ie, socially constructed roles) on TCP admission, length of stay, and services received. We will also examine how living in rural or remote communities may influence one's ability to access TCPs. The analysis will be conducted by the investigator members of the team in collaboration with representatives of the knowledge users and stakeholder groups.

\section{Step 6: consultation and knowledge translation}

Consistent with the principles of the collaborative/participatory approach to research, we formed the research team to include different stakeholder groups (researchers, family caregiver, clinical and policy decision makers). The stakeholder groups were involved in focusing the research question and selecting the methodology. In addition, we will engage them in the data abstraction, analysis of the data, and interpretation of findings. This will be facilitated through regularly scheduled meetings and communication. Lastly, we will employ both integrated knowledge translation (KT) and end-of-grant KT strategies ${ }^{26}$ inviting stakeholder groups to participate in dissemination of the findings (through presentations to local clinical and policy audiences and preparation of manuscripts and policy briefs). The overarching goal of our KT plan is to build a robust evidence base to support the development of person-centred and integrated care and service delivery, to promote optimal functioning and improvement in the quality of life of older adults and their caregivers. Once data are analysed, we will hold a stakeholder consultation workshop to present preliminary findings to the team to solicit feedback on the final content before broader dissemination and endof-grant KT activities. End-of-grant KT activities will include the development of fact sheets, evidence briefs, and reports targeted at specific audiences; conference presentations and publication of results in peer-reviewed journals.

\section{ETHICS AND DISSEMINATION}

This ScR does not require ethics approval. Our team comprises researchers, clinicians, caregivers and representatives from the healthcare system at local, regional, and provincial levels, who are all target users of our findings. Thus, we begin this project with the support and commitment of numerous knowledge users, who are keen to use and disseminate our findings. Upon study completion, we will disseminate reports targeted at specific audiences. In addition, our knowledge user members are affiliated 
with local, provincial, national and international research networks, clinical-care organisations and stakeholder groups. Therefore, we will also disseminate findings to and through these organisations, benefiting from their existing KT mechanism and platforms. Scientific findings from the review will be presented at national and international conferences and published in peer-reviewed interdisciplinary journal.

One of the key strengths of this project is the collaborative and participatory approach to research undertaken by our highly skilled team that also includes family caregivers and multidisciplinary and multi-sectoral partners from across Ontario. The considerable experience of our team members, and the unique perspectives and priorities they bring to this project ensure that the research findings will meaningfully impact the development and implementation of effective TCPs.

\section{Author affiliations}

${ }^{1}$ Research, Toronto Rehabilitation Institute-University Health Network, Toronto, Ontario, Canada

'Lawrence S. Bloomberg Faculty of Nursing, University of Toronto, Toronto, Ontario, Canada

${ }^{3}$ Institute for Clinical Evaluative Sciences, Toronto, Ontario, Canada

${ }^{4}$ North East Local Health Integration Network, Sudbury, Ontario, Canada

${ }^{5}$ Research Institute, Health Sciences North, Sudbury, Ontario, Canada

${ }^{6}$ Visiting Homemakers Association Home Healthcare, Toronto, Ontario, Canada

${ }^{7}$ Dalla Lana School of Public Health, University of Toronto, Toronto, Ontario, Canada

${ }^{8}$ Ontario Ministry of Health and Long-Term Care, Toronto, Ontario, Canada

${ }^{9}$ Policy and Research, Canadian Association of Retired Persons (CARP), Toronto,

Ontario, Canada

${ }^{10}$ Institute of Health Policy Management and Evaluation, University of Toronto,

Toronto, Ontario, Canada

${ }^{11}$ Daphne Cockwell School of Nursing, Ryerson University, Toronto, Ontario, Canada

\section{Twitter Katherine S McGilton @Kathy_mcgilton}

Contributors All authors (KSM, SV, JBabineau, JBethell, SEB, EB, MK, JEM, SM, KN, AO, MTEP, AS, LTW, WPW and SS) have been involved in the design of the study, acquisition analysis and interpretation of data, drafting manuscript, critically revising the manuscript for important intellectual content and approval of final version; as well agree to be accountable for all aspects of the work. Author KSM was also involved in the conception of the study. We thank family caregiver, Margaret Keatings for her interest and guidance during the design and the conduct of this scoping review.

Funding This work is supported by the Canadian Institutes of Health Research (CIHR) Operating Grant: Transitions in Care - Best and Wise Practices Grants (ClHR Funding Number BWP-163068). KSM is supported by the Walter and Maria Schroeder Institute for Brain Innovation \& Recovery.

Competing interests None declared.

Patient consent for publication Not required.

Provenance and peer review Not commissioned; externally peer reviewed.

Open access This is an open access article distributed in accordance with the Creative Commons Attribution Non Commercial (CC BY-NC 4.0) license, which permits others to distribute, remix, adapt, build upon this work non-commercially, and license their derivative works on different terms, provided the original work is properly cited, appropriate credit is given, any changes made indicated, and the use is non-commercial. See: http://creativecommons.org/licenses/by-nc/4.0/.

\section{ORCID iDs}

Katherine S McGilton http://orcid.org/0000-0003-2470-9738

Shirin Vellani http://orcid.org/0000-0001-6032-0266

Jennifer Bethell http://orcid.org/0000-0002-6141-9011
Walter P Wodchis http://orcid.org/0000-0003-4738-8447

Souraya Sidani http://orcid.org/0000-0002-9115-2389

\section{REFERENCES}

1 Marengoni A, Rizzuto D, Wang H-X, et al. Patterns of chronic multimorbidity in the elderly population. J Am Geriatr Soc 2009;57:225-30.

2 Costa AP, Poss JW, Peirce T, et al. Acute care inpatients with longterm delayed-discharge: evidence from a Canadian health region. BMC Health Serv Res 2012;12:172.

3 Landeiro F, Roberts K, Gray AM, et al. Delayed Hospital discharges of older patients: a systematic review on prevalence and costs. Gerontologist 2019;59:e86-97.

4 Barrette PA. 1,005 delayed days: a study of adult psychiatric discharge. Psychiatric Services 1981;32:266-8.

5 Halliday HL, Grant AP. Social factors associated with delay in discharge from an acute medical ward. Ulster Med 1975;44:139-44.

6 Canadian Institute for Health Information. Definitions and guidelines to support ALC designation in acute inpatient care introduction guidelines to support ALC designation by clinicians, 2016. Available: https://www.cihi.ca/sites/default/files/document/acuteinpatientalcdefinitionsandguidelines_en.pdf

7 Health Quality Ontario (HQO). Measuring up 2018: a yearly report on how Ontario's health system is performing, 2018. Available: https:// www.hqontario.ca/Portals/0/Documents/pr/measuring-up-2018-en.pdf

8 Rojas-García A, Turner S, Pizzo E, et al. Impact and experiences of delayed discharge: a mixed-studies systematic review. Health Expectations 2018;21:41-56.

9 Bender D, Holyoke P. Why some patients who do not need hospitalization cannot leave: A case study of reviews in 6 Canadian hospitals. Healthc Manage Forum 2018;31:121-5.

10 Kuluski K, Im J, McGeown M. "It's a waiting game" a qualitative study of the experience of carers of patients who require an alternate level of care. BMC Health Serv Res 2017;17:318.

11 McCloskey R, Jarrett P, Stewart C, et al. Alternate level of care patients in hospitals: what does dementia have to do with this? Can Geri J 2014;17:88-94.

12 Coleman EA, Boult C, American Geriatrics Society Health Care Systems Committee. Improving the quality of transitional care for persons with complex care needs. J Am Geriatr Soc 2003;51:556-7.

13 Wee S-L, Loke C-K, Liang C, et al. Effectiveness of a national transitional care program in reducing acute care use. J Am Geriatr Soc 2014;62:747-53.

14 Colquhoun HL, Levac D, O'Brien KK, et al. Scoping reviews: time for clarity in definition, methods, and reporting. J Clin Epidemiol 2014:67:1291-4.

15 Arksey H, O'Malley L. Scoping studies: towards a methodological framework. Int J Soc Res Methodol 2005;8:19-32.

16 Levac D, Colquhoun H, O'Brien KK. Scoping studies: advancing the methodology. Implement Sci 2010;5.

17 Daudt HML, van Mossel C, Scott SJ. Enhancing the scoping study methodology: a large, inter-professional team's experience with Arksey and O'Malley's framework. BMC Med Res Methodol 2013;13:48.

18 Tricco AC, Lillie E, Zarin W, et al. PRISMA Extension for Scoping Reviews (PRISMA-ScR): checklist and explanation. Ann Intern Med 2018;169:467-73.

19 Bramer WM, Giustini D, de Jonge GB, et al. De-duplication of database search results for systematic reviews in endnote. $J$ Med Libr Assoc 2016;104:240-3.

20 Covidence. Systematic review software. Melbourne, Australia.

21 Davis R. Voices of Native Hawaiian kupuna (elders) living with chronic illness: "knowing who I am". J Transcult Nurs 2010;21:237-45.

22 Waugh E, Mackenzie L. Ageing well from an urban Indigenous Australian perspective. Aust Occup Ther J 2011;58:25-33.

23 Cooke M, Mitrou F, Lawrence D, et al. Indigenous well-being in four countries: an application of the UNDP's human development index to Indigenous peoples in Australia, Canada, New Zealand, and the United States. BMC Int Health Hum Rights 2007;7:9.

24 Commisso E, McGilton KS, Ayala AP, et al. Identifying and understanding the health and social care needs of older adults with multiple chronic conditions and their caregivers: a protocol for a scoping review. BMJ Open 2017;7:e0182470.

25 Donabedian A. Evaluating the quality of medical care. Milbank $Q$ 2005;83:691-729.

26 Canadian Institutes of Health Research. Guide to knowledge translation planning at CIHR: integrated and End-of-Grant approaches, 2012. Available: http://www.cihr-irsc.gc.ca/e/ documents/kt_Im_ktplan-en.pdf 